\title{
Verre cassé d'Alain Mabanckou à la recherche d'un lecteur (modèle)
}

\author{
Verre cassé by Alain Mabanckou in Search \\ of a (Model) Reader
}

Petr Vurm [vurm@phil.muni.cz]

Masarykova univerzita, République tchèque

\section{RÉSUMÉ}

Dans le présent article, nous aborderons quelques modalités de la dynamique entre l'auteur, le narrateur et le lecteur dans une perspective narratologique. Pour ce faire, nous allons analyser le roman Verre cassé d'Alain Mabanckou, qui est un exemple d'une écriture francophone globalisée et hybride. Plus précisément, nous allons nous pencher sur le rôle d'un lecteur actif, «complice » du narrateur, qui accepte le défi et le plaisir de lecture, ainsi que le jeu de déchiffrage du roman en question, truffé de références intertextuelles. Et plus particulièrement encore, en parlant de ce roman, dont la lecture superficielle est plutôt facile, qui en cache pourtant une lecture profonde et profondément littéraire, nous partirons à la recherche du lecteur-modèle du texte.

\section{MOTS-CLÉS}

Mabanckou ; Verre cassé ; narrateur ; lecteur ; lecteur-modèle ; intertextualité ; hybridité ; mondialisation

\section{Abstract}

In this article, we will address several aspects of the dynamics between author, narrator and reader. To accomplish this goal, we would like to analyze the novel Verre cassé by Alain Mabanckou, who is an example of globalized and hybrid Francophone writing. More precisely, we shall examine the role of the active reader, a narrator's "accomplice", who accepts the challenge and the pleasure of reading, or rather the play of deciphering a novel full of intertextual references. And still more in particular, by a close reading of this novel, the superficial reading of which is rather easy, but which is hiding a profound and deeply literary reading level, we shall look for the model reader of the text.

\section{KEYWORDS}

Mabanckou; Verre cassé; narrator; reader; model reader; intertextuality; hybridity; globalization

REÇU 2019-06-23; ACCEPTE 2019-08-12

Cet article est le résultat d’une recherche dans le cadre de la bourse GA ČR du projet 17-26127S: « Nouveaux narratifs, nouvelles esthétiques: les auteurs contemporains de l'Afrique francophone du point de vue de l'hybridité, des migration et de la globalisation mondiale » ("New Narratives, New Aesthetics: Contemporary Francophone African Authors Through Hybridity, Migration and World Globalization »). 
Paru aux éditions du Seuil, le roman Verre cassé a remporté plusieurs prix littéraires dont le Prix Ouest-France/Étonnants Voyageurs, le Prix des Cinq Continents de la Francophonie et le Prix du livre RFO. Il a été finaliste du prix Renaudot 2005, prix que son auteur a reçu une année plus tard avec Mémoires de porc-épic, également paru au Seuil. En 2012, le quotidien anglais The Guardian a désigné ce roman comme l'un des 10 meilleurs livres africains contemporains.

Nous pouvons peut-être contester cette dernière constatation, cependant il est indéniable que Verre cassé est le roman de Mabanckou qui a eu le plus grand succès dans le monde, traduit en une dizaine de langues, mis en scène plusieurs fois, c'est celui qui l'a rendu vraiment célèbre. L'une des explications du succès du roman tient sans doute à son caractère universel ou au moins universalisant, débordant du contexte de la littérature francophone subsaharienne, roman qui en quelque sorte toucherait la communauté des lecteurs toute entière. Nous croyons, avec Umberto Eco, Hans-Robert Jauss et Wolfgang Iser, qu'un lecteur actif, complice, pratiquant une lecture complice y est pour quelque chose. Or, dans notre article, nous voudrions nous pencher sur le rôle de ce lecteur actif, complice qui accepte le défi et le plaisir de la lecture, ou plutôt le jeu de déchiffrage du roman plein de références intertextuelles. Plus particulièrement, en parlant de ce roman dont la lecture superficielle est plutôt facile, qui en cache pourtant une lecture profonde et profondément littéraire, nous souhaitons chercher le lecteur-modèle du texte. Ce lecteur, selon Eco, est un lecteur capable de s'approprier les codes de l'émetteur, de l'auteur ou du narrateur de Verre cassé. Rappelons ce prototype du lecteur abordé dans Lector in fabula de Umberto Eco:

Chaque destinataire se trouvant confronté à un texte, se retrouve ainsi confronté en premier lieu à sa surface, à sa manifestation extérieure et linguistique, et doit actualiser toute une série de chaînes d'artifices. Puisqu'il est à actualiser, un texte est donc incomplet. (Eco 1985 : 28)

Umberto Eco stipule que le texte représente un «tissu de non-dit » : « non-dit », explique-t-il, « signifie non manifesté en surface, au niveau de l'expression » (Eco 1985 : 29). C'est justement ce « non-dit » qui demande à être actualisé. Pour permettre cette actualisation, le lecteur doit produire une série de mouvements coopératifs et conscients. Or, nous croyons que Verre cassé est précisément un texte ouvert selon Eco, invitant à de nombreuses analyses narratologiques ainsi qu'à des jeux dynamiques entre l'auteur, le narrateur, le lecteur. Le texte qui n'est jamais clos. Avant de nous plonger au " cœur des ténèbres ", ou plutôt, parmi les "tessons du verre cassé ", rappelons brièvement l'histoire du roman.

Cinquième roman d'Alain Mabanckou, Verre cassé apparaît comme un conte « horrifique ». Ecrit en une seule phrase, presque d'un seul souffle, il raconte la vie telle qu'elle se déroule dans la majorité des périphéries africaines. C’est un récit qui se veut «la bibliothèque » des récits biographiques d’une catégorie d'individus souvent marginaux de l'Afrique et du Congo-Brazzaville en particulier. L'auteur congolais met en scène des personnages en situation d'immigration dans leur milieu d'origine pour nous permettre de saisir la problématique de l'identité floue et hybride des Africains contemporains, au sein même de leur propre espace géographique. Verre Cassé est aussi le nom du personnage princi$\mathrm{pal}^{1}$. Il a pour mission d'immortaliser les histoires sensationnelles des clients fidèles du bar « Le crédit

1 Par souci de clarté, nous distinguons dans notre texte entre Verre cassé, titre du roman, et Verre Cassé, nom du personnage. 
a voyagé ». Le propriétaire lui confie cette mission, compte tenu de ses capacités intellectuelles distinguées. Comme son nom l'indique, Verre Cassé est un personnage explosé, fracassé, qui se trouve en quête d'une identité nouvelle, comme le héros le dit lui-même : "a-t-on jamais vu un verre cassé être réparé ». La particularité de l'univers romanesque de Mabanckou est qu'il met en évidence des sujets ayant pour dénominateur commun la déchéance. Sans se limiter à cette "dépression » identitaire, se déploie dans la suite de ce déclin un manifeste de devenir autre, c'est-à-dire la redéfinition de sa propre identité. Pour ce faire, le narrateur se met à l'écart des conventions socioculturelles africaines, qu'il se propose de revisiter. Allant dans son récit de «l'homme aux Pampers » dégradé physiquement à cause d'une fausse révélation de son épouse, à «l'imprimeur » psychologiquement atteint, passant par la narration autobiographique du « verre cassé » dont l'histoire parle d'un homme qui jadis fut brillant instituteur, devenu aujourd'hui un buveur invétéré, on peut lire ici une déflagration identitaire au cœur de la trame narrative. C’est une catégorie de personnages fort marginalisés par rapport à la société congolaise. Ce particularisme est marqué par la chute réelle et métaphorique de ces derniers, dont le bar symbolise la décadence.

D'ailleurs, nous ne savons pas exactement où se situe le bar "Crédit a voyagé » sinon qu’on peut le trouver sur l'avenue de l'Indépendance et qu'il n'est pas loin de la rivière Tchinouka, qui coule à Pointe-Noire ; les deux quartiers qui sont nommés, le Quartier Trois-Cents et le Quartier Rex, sont eux aussi des quartiers de Pointe-Noire. Cependant le bar «Crédit a voyagé » renvoie moins à un lieu réel qu'à l'univers littéraire et à son intertextualité, réclamant de cette manière un lecteur actif, puisque le nom du bar renvoie avant tout à l'univers de Louis-Ferdinand Céline. De cette façon, Mabanckou semble se réclamer de l'aspect novateur et choquant de Céline, à cause de sa langue verte - langage oral, populaire, argotique, d'une violence crue, dont se servent les narrateurs dans le but de dénoncer la misère sociale, la guerre, le colonialisme.

\section{Le cahier - entre un objet banal et une oeuvre littéraire}

L'écrit, selon le patron du bar «Crédit a voyagé », est plus fiable que l'oral pour la conservation de la mémoire, ainsi celui-ci souhaite qu’une trace écrite de son bar subsiste. Verre Cassé-narrateur est chargé de mener à bien cette tâche. Bien que cela ne soit pas mentionné explicitement au début du roman, on imagine aussitôt que le texte qu'on lit est celui que Verre Cassé a rédigé dans le cahier reçu, sans doute parce qu'il s'exprime à la première personne. De plus, l'intitulé " premiers feuillets », sur lequel le récit s'ouvre, évoque fortement un cahier. L'ambiguïté entre le livre que nous lisons et le cahier supposé avoir été rédigé par le personnage est d'emblée manifeste : le patron du bar prétend qu'il conservera ce cahier pour son seul usage, cependant il croit dur comme fer que Verre Cassé est capable de créer un livre, cela sous-entend que la publication du cahier a été envisagée dès le début. Par la suite, c'est d'une façon extrêmement cohérente que se développe toute une stratégie, dont la finalité est d'amener le lecteur à croire en ce dispositif du cahier composé par le personnage, procédé par lequel la prise de parole de Verre Cassé s'explique, se trouve être légitimée, tout en justifiant la forme sous laquelle le roman se présente.

L'existence du cahier en tant qu'objet matériel, visible, palpable, est rappelée tout au long du récit. Verre Cassé demande à l'imprimeur pourquoi ce dernier veut lui raconter son histoire, et il relate que : 
L'Imprimeur s'est mis à réfléchir, le regard vers le ciel, «il paraît même que tu écris quelque chose sur les types bien de ce bar, tu écris ça dans un cahier, ça doit être ce cahier qui est à côté de toi, n'est-ce pas ", je n'ai pas répondu, j'ai posé une main sur la page du cahier parce que le type tentait de lire mes gribouillis, j’aime pas ça. $(63)^{2}$

Verre Cassé refuse que le type aux Pampers, soudain mécontent que son récit y soit inscrit, s'empare du cahier : " je me suis levé, j'ai mis le cahier sur le tabouret et me suis assis dessus, comme ça il ne pouvait pas me l'arracher de force. » (219-20). Les deux se battent, se réconcilient, et Verre Cassé conclut : et j'ai ramassé mon cahier qui traînait par terre pour aller faire un tour dans le quartier. » (224).

L'activité de l'écriture est mise en scène et racontée à de nombreux endroits. Verre Cassé semble se plier aux exigences de l'Imprimeur, qui veut le « voir écrire » pendant qu'il parle :

$[\ldots]$ «bon, je vais commencer par le début, mais écoute-moi bien parce que ce je vais te raconter est très important, prends note, prends bien note, je veux te voir écrire quand je parle, » et pendant que je griffonnais quelques-uns de ses premiers mots, il a dit... (65).

Verre Cassé griffonne-t-il ou note-t-il réellement ce que dit l'Imprimeur ? Celui-ci, après avoir parlé un moment, s'inquiète : "j'espère que tu notes bien ce que je te raconte depuis un moment, hein, ... » (74). L'Imprimeur paraît rassuré et poursuit son histoire. Il est difficile de croire que le cahier soit véritablement le résultat d'une suite de notes prises sur le vif dans le bar, au hasard des rencontres, car le récit apparaît doté d'une structure temporelle complexe. On y trouve en alternance des récits rétrospectifs rédigés essentiellement au passé composé et à l'imparfait, les discours rapportés des personnages, et des notations au présent qui rendent compte du moment où Verre Cassé écrit, ou encore des passages au futur, où Verre Cassé imagine ce qu'il fera une fois le cahier achevé :

Voyons, en tout cas, je dois me reposer, ne plus écrire une seule ligne, ne rien relire, je continuerai alors plus tard, je ne sais pas quand, mais je continuerai, je ne tiens pas à consacrer toute mon énergie à ça, et quand j'aurai terminé la deuxième partie, je m'en irai, je m’en irai loin, je ne sais pas où, mais je m'en irai, je m'en fous de ce que pensera L'Escargot Entêté, mais je serai loin, loin du Crédit a voyagé. (109)

Verre Cassé projette de quitter le bar dès que la rédaction de son cahier sera achevée. A la deuxième page de son récit, il laisse déjà entrevoir ce programme : «mais quand il lira tout ça je ne serai plus client de son bar, j'irai traîner mon corps squelettique ailleurs, je lui aurai remis le document à la dérobée en lui disant " mission terminée » (12). Une fois le cahier refermé, Verre Cassé semble se réduire à son squelette, comme si à partir du moment où il renonçait à écrire, il perdait sa «substance ». De cette façon, il est mis en scène autant qu'il se met en scène lui-même comme existant essentiellement dans le texte du roman et par la fiction qui le fait naître. De plus, à la fin, il déclare que sa place est désormais « dans l'autre monde » (246). Ainsi, la frontière qui sépare la fiction de la réalité se révèle très incertaine. 


\section{Ambiguïté auteur/personnage/lecteur}

La façon dont Verre Cassé décrit comment lui apparaissent les clients du bar est singulière : «j’ai rencontré L'Imprimeur comme je rencontre souvent la plupart des nouveaux personnages de ce bar, ils sortent de je ne sais où, et les voilà devant moi » (61). Il est rare d'employer le terme personnage pour un être humain réel, à moins que ses traits n'évoquent justement un personnage de fiction. Soit Verre Cassé se trouve dans un lieu fréquenté par des gens dont les traits de caractère sont tels qu'ils évoquent davantage des personnages de fiction que des personnes réelles, soit les personnages qu'il décrit surgissent devant lui par la force de son imagination et ne sont effectivement que des personnages de fiction. Ainsi, les deux personnages principaux, l'Escargot entêté et Verre Cassé, peuvent être considérés à la fois comme des auteurs, des personnages et des lecteurs, ce qui offre de riches pistes interprétatives. Observons leur onomastique et leur fonction dans le récit. Commençons par l'Escargot entêté.

Le patron du bar a dû faire preuve d'obstination face au mouvement d'opposition qui a suivi l'ouverture de son établissement, ce qui justifie qu'on le qualifie d'entêté. Quant au surnom Escargot, c'est sans doute une référence au roman de Rachid Boudjedra, dans lequel le narrateur, un fonctionnaire solitaire, frustré et maniaque, s'imagine qu'un escargot s'entête à le persécuter.

L'Escargot entêté est à la fois le propriétaire du bar mais aussi est-il le premier, et selon le propos initial, le seul lecteur du cahier. Son entêtement tient également d'une (méta)critique littéraire d'un lecteur prétendument naï :

J'avais pas bien vu, mais c'est vraiment le désordre dans ce cahier, y a pas de points, y a que des virgules et des virgules, parfois des guillemets quand les gens parlent, c'est pas normal, tu dois mettre ça un peu au propre, tu crois pas, hein, et comment moi je peux lire tout ça si c'est collé comme ça, faut laisser encore quelques espaces, quelques respirations, quelques moments de pause, tu vois, j’attendais quand même mieux de toi, je suis un peu déçu, excuse-moi, ta mission n'est pas encore terminée, tu dois recommencer. (27)

Rappelons aussi que les goûts littéraires de l'Escargot entêté sont bien raffinés et aux antipodes de la création de Verre Cassé : par exemple, puisqu'il aime beaucoup La Mort du Loup de Vigny, il récite sans cesse ce poème. Le nom de Verre Cassé, renvoie d'abord vers tout ce qui est fragmentaire, brisé, impossible à recomposer, comme l'est le caractère du narrateur lui-même. De surcroît, sur un autre niveau d'intertextualité, purement africaine, Verre cassé est le titre d'une chanson populaire de Simaro Lutumba, un chanteur congolais qui chante en lingala. Selon Abel Kouvouama

[...] ses thèmes de prédilection [de Lutumba] sont le divorce, la stérilité, les amours ratées qu’il met en musique avec subtilité, accompagnée d’une certaine mélancolie teintée d’amertume. Mais il chante également la nature, le bonheur que vivent les gens pauvres en dépit de la misère du monde. (Kouvouama 2013 : 101)

Le goût pour la culture populaire est aussi affiché par Verre Cassé lorsqu'il évoque ses personnages de bande-dessinée préférés ou lorsqu'il mentionne ses romans préférés de San Antonio. 
Verre Cassé peut aussi être une référence au roman Le cassé de l'auteur québécois Jacques Renaud, que Jean-Marc Moura cite comme exemple d'un recours à une langue orale fictive, ce qui correspondrait au style de Mabanckou. Or, par les intertextes variés et assez hétérogènes, Verre Cassé revendique ses liens avec la culture et les parlers populaires, mais aussi avec les gens qui, comme lui, ne parviennent pas à s’adapter aux exigences de la société «normale », voire des élites.

\section{Le lecteur dans le labyrinthe de l'intertextualité}

Selon Bakhtine, tout discours est un « lieu où se croisent, se rencontrent et se séparent des points de vue différents, des visions du monde, des tendances » (Bakhtine 1984 : 301), un lieu où se manifeste la diversité sociale. Ainsi, la notion d'intertextualité fonctionne dans la perspective du dialogisme, de telle sorte qu'au modèle vertical, celui de la tradition et de la filiation se substitue, selon Natalie Piégay-Gros, « le modèle horizontal de l'échange avec le langage environnant» (2002:15).

Verre cassé est un roman intrinsèquement intertextuel, à tel point que nous pouvons nous demander ce qui en resterait si l'on enlevait toutes les références intertextuelles. De par sa composition, il réclame donc un lecteur actif, capable de reconstituer les non-dit et déchiffrer les nombreux clins d'œil.

Or, Verre cassé est un roman qui, selon Oana Panaïé, « cannibalise les discours les plus divers : littéraire, scientifique, administratif, juridique, commercial ", démontre qu'en effet un texte peut être conçu comme une combinatoire d'éléments hétérogènes, pour autant que soit effectué « un travail de transformation et d'assimilation » de ces fragments " par un texte centreur qui garde le leadership du sens » (Panaïté 2012 : 177). Pour ne citer qu'un seul exemple :

Et puis il y a eu une action mystique des gardiens de la morale traditionnelle, des chefs de tribu avec leurs gris-gris qu'ils jetaient à l'entrée de l'établissement, avec leurs paroles de malédiction qu'ils adressaient au patron du Crédit a voyagé, avec des âmes mortes qu'ils faisaient parler, et ils prophétisaient que le commençant allait crever à petit feu, qu'ils allaient le pousser doucement à prendre lui-même un ascenseur pour l'échafaud, mais en vain aussi, et puis il y a eu enfin une action directe de ceux qui regrettaient la joie de mener une vie de boy, et alors ces gens de bonne réputation ont tendu un piège sans fin au patron avec leurs casseurs cagoulés qui sont venus au coeur des ténèbres. (14-15)

Nous avons pu identifier dans cet extrait au moins sept références intertextuelles et interculturelles - à Gogol, à un film de Louis Malle, à l'Action directe (groupe français de militants et terroristes anarchiques), à Une vie de boy de F. Oyono, à Un piège sans fin roman d'Olympe Bhély-Quénum, Au cœur des ténèbres est un roman de Joseph Conrad et à la $L a$ bonne réputation, un film muet de Pierre Marodon. Plus loin, on parle de vraies "belles du Seigneur ", jamais un homme ne vivra de telles "stupeurs, de tels tremblements ", sous son toit conjugal, elle a dit que même mort elle me piétinerait, qu'elle " irait cracher sur ma tombe ", elle a dit que je ressemblais à un marin rejeté par la mer. Comme nous pouvons le constater à partir de ce torrent de références intertextuelles, Verre Cassé dépasse la demande 
de l'Escargot entêté qui voulait simplement un témoignage sur l'existence du bar, « un livre qui parlerait de nous ici ». La visée principale de l'écriture de Verre Cassé est plutôt de faire s'entrecroiser dans son récit un maximum d'échos provenant d'autres textes, d'autres discours. De cette façon, comme nous l'avons déjà indiqué, le texte cannibalise ses architextes, ses ancêtres symboliques, il tend à se libérer du monde réel pour s'établir pleinement au sein du jeu de la référence intertextuelle.

La diversité et la surabondance de références intertextuelles proposées dans Verre Cassé est déconcertante. Sachant qu'il est possible de se référer à un titre de livre sans l'avoir lu, on se demande si chaque titre évoqué dans Verre Cassé renvoie véritablement au contenu et à la signification de l'œuvre, ou seulement à ce que l'œuvre peut superficiellement évoquer, par exemple un genre, une époque, un mouvement ou une région géographique. On soupçonne souvent une gratuité associative chez Mabanckou, une mécanique plutôt qu'une logique, dans le sens où les titres des oeuvres connues fonctionnent comme des expressions figées dans la tête du narrateur plutôt que de véritables références intertextuelles et contextuelles.

Cela témoigne du fait que Verre Cassé-personnage représente le premier lecteur du récit, celui qui lit, fait le choix et le tri finals. C'est lui qui a été le "grand assidu des bibliothèques ", qui a dans sa jeunesse effectué un parcours initiatique en tant que lecteur mais qui par la suite doute de ce qu'aurait pu lui apporter l'étude de la littérature : « il est vrai que je n’aurais pas pu aller loin avec la littérature si j’avais fait de longues études, ça ne mène nulle part la littérature » (208).

Or, Verre Cassé représente un lecteur fictif intra-diégétique par excellence, qui a fait un voyage littéraire d'apprentissage :

[...] il fallait vraiment partir, remonter vers le nord du continent, vivre la plus haute des solitudes, pénétrer dans le village de Macondo, y vivre cent ans de solitude, d'aventures, de découvertes, se laisser fasciner par les contes d'amour de folie et de mort, passer en toute discrétion par le tunnel qui mène vers la connaissance des sentiments humains (210-11).

Mais si la lecture a d'abord distrait et éduqué Verre Cassé, la lassitude l'a peu à peu gagné : " la volonté s'est émoussée au fil des ans, et je me laisse aller comme une immondice qui suit le courant d'un fleuve détourné » (213), dit-il.

Le Verre cassé-roman est en quelque sorte le résultat des lectures, voire un journal de lecture de Verre Cassé-personnage. Dans sa liste, toutes les oeuvres sont placées sur le même plan. La force d'évocation des titres est déployée de façon magistrale, et les univers que ces titres font surgir apparaissent concrètement présents, presque animés, comme dans « ceux qui fabriquent des cérémonies pour danser la polka » (213), une phrase-valise résumant deux oeuvres de Kossi Efoui, autre écrivain contemporain, originaire du Togo, à savoir La Fabrique des cérémonies et La Polka.

Or, le texte tout entier indique l'ouverture du champ de lecture localisé au Congo vers la littérature-monde, par analogie à la transition du destinataire à partir de l'Escargot entêté vers le lecteur-modèle généralisé. 


\section{Conclusion}

Dans Verre cassé d'Alain Mabanckou, il ne s'agit pas d'inventer une nouvelle formule d'écriture et de lecture, loin de là. Il s'agit d'en trouver une faisant déjà partie de la mémoire collective et de la «mémoire intertextuelle ", qui prendrait juste un sens neuf du fait du changement de contexte dans un monde globalisé et hybride. En réaction à ce changement, le texte mabanckouien, par son jeu intertextuel et par sa stratégie narrative, semble souligner le fait que tout a déjà été dit, que rien de neuf ne peut plus surgir et que la seule possibilité serait de revenir aux textes du passé et de les recycler d'une manière créative.

Pour répondre à la question initiale, à savoir qui serait en fin de compte le lecteur modèle ou lecteur empirique du roman et comment ce roman prévoirait-il son lecteur ? Nous croyons avoir démontré que le roman prévoit son lecteur à plusieurs niveaux : il y a plusieurs lecteurs fictifs, Verre Cassé, l'Escargot entêté, et les clients du bar. De surcroît, le roman envisage bien sûr son lecteur dans le monde réel. Il s'agirait d'un lecteur actif, complice, qui serait au courant de la francophonie littéraire et de ses problèmes. Une amorce de réponse quant au lecteur-modèle a été donnée sur un ton d'ironie caustique par Mabanckou lui-même :

J'écris en français pour que l'on continue à me poser sempiternellement la question « Pourquoi écrivez-vous en français ? » [...] J'écris en français pour manger dans tous les râteliers de l'institution francophone. [...] J’écris en français parce que je voulais être publié, primé - dis-je ! -, célébré à Paris.

[...] J'écris en français parce que je rêve de recevoir un jour le prix Goncourt. J'écris en français parce que je rêve de traîner mes vieux os jusqu'au Quai Conti [...], (journal Libération, 16 mars 2006)

Or, se pourrait-il que Mabanckou auteur empirique verrait d'abord les lecteurs modèles parmi ceux de l'Académie française ou de l'Académie Goncourt? A savoir, chers lecteurs !

\section{Références bibliographiques}

Bakhtine, M. (1984). Esthétique de la création verbale. Paris: Seuil.

Cazenave, O. (2004). Afrique sur Seine : une nouvelle génération de romanciers africains à Paris. Paris: L'Harmattan.

Eco, U. (1985). Lector in fabula. Paris: B. Grasset, collection Figures.

Gaetzi, C. (2015). L'art de déjouer le témoignage par la fiction. Verre cassé d'Alain Mabanckou. Paris : Archipel.

Kouvouama, A. (2013). Anthropologie de la chanson congolaise de variétés. Paris: Paari éd.

Libération (2006). Paris: Libération SARL.

Mabanckou, A. (1998). Bleu Blanc Rouge. Paris: Présence africaine. . (2009). Black bazar. Paris: Seuil. 
(2005). Verre cassé. Paris: Seuil.

Moudileno, L. (2006). Parades postcoloniales. La fabrication des identités dans le roman congolais. Paris: Carthala.

Panaïté, O. (2012). Des littératures-mondes en français : écritures singulières, poétiques transfrontalières dans la prose contemporaine. Amsterdam - New York: Rodopi.

Pannenborg, W. A. (1955). Écrivains satiriques: caractère et tempérament étudiés d'après des données empruntées à des biographies et à d'autres enquêtes. Paris: PUF.

Piégay-Gros, N. (2002). Introduction à l'intertextualité. Paris: Nathan.

Sayad, A. (1977). Les trois " âges » de l'émigration algérienne en France. Actes de la recherche en sciences sociales, $15,59-79$.

Simédoh, V. (2012). L'Humour et l'ironie en littérature francophone subsaharienne. Des enjeux critiques à une poétique du rire. New York: Peter Lang. 
